\title{
PROPAGACIÓN VEGETATIVA in vitro A PARTIR DE HOJAS JÓVENES DE CAÑA DE AZÚCAR (Saccharum officinarum L.) ${ }^{1}$
}

\author{
José M. Cuéllar ${ }^{2}$
}

\begin{abstract}
RESUMEN
Propagación vegetativa in vitro a partir de hojas jóvenes de caña de azúcar (Saccharum officinarum $L$.) .Se evaluaron la respuesta de dos variedades de la caña de azúcar: Mex 70-485 y Mex 68-P-23, en cada una de las etapas de la propagación in vitro. Se utilizaron hojas jóvenes aún enrolladas. Se inocularon explantes de alrededor de $5 \mathrm{~mm}$ de longitud en medio de Murashige y Skoog (1962) modificado para cada una de las etapas. Para la callogénesis se utilizaron $3,0 \mathrm{mg} / \mathrm{l}$. de 2,4-D, para el crecimiento y desarrollo de "callos" 2,$0 ; 2,5$ y 3,0 mg/l. de 2,4-D y para la diferenciación no se uso regulador. Para la rizogénesis se utilizaron varios medios: un medio con la mitad de la concentracción de macronutrimentos, otro con la concentracción total de las sales y otro con las sales completas suplementado con $5 \mathrm{mg} / \mathrm{l}$ de AIA. En la fase de formación de callo, el mayor problema fue la fenolización de los explantes. La mejor concentración de ácido 2,4-D fue de 3,0 mg/l , tanto para la formación de callo como para su crecimiento y desarro1lo; La diferenciación se dio con la sustracción de ácido 2,4-D del medio, y la rizogénesis fue favorecida con el suplemento de $5 \mathrm{mg} / \mathrm{l}$ de AIA.
\end{abstract}

\begin{abstract}
In vitro test for the vegetative propagation of sugar cane (Saccharum officinarum $L$.) from young leaves.This paper reports on the first in vitro cultivation tests done in El Salvador. The responses of cultivars MEX 70-485 and MEX 68-P-23 on various propagation stages, were evaluated in vitro. Young leaves still in the rolled stage were utilized. Explants 5 $\mathrm{mm}$ in lenght were placed on a Murashige and Skoog (1962) medium, modified for each stage of planttet recovery as follows: For the development and growth of "calluses" dosis, $2.0,2.5$ and $3.0 \mathrm{mg} / \mathrm{l}$ of $2.4 / \mathrm{D}$ were added and for differentiation, it was subtracted. To induce rhyzogenesis we used a medium with half of the macro-nutrients, one with the complete salts the other with the complete salts plus a supplement of $5 \mathrm{mg} / \mathrm{l}$ of AIA. Outstanding results were the following: In the phase of "calluses" formation, the major problem was the phenolization of the explants; the best 2.4-D acid concentration was $3.0 \mathrm{mgl}$, for both the calluses formation as for their growth and development. The differentiation was obtained with the renoval of 2.4-D acid from the medium, and the rhyzogenesis was favored with the supplement of $5 \mathrm{mg} / \mathrm{l}$ ofAIA
\end{abstract}

\section{INTRODUCCIÓN}

La Caña de Azúcar junto al Café, son los cultivos agroindustriales más importantes de El Salvador, generadores de empleo y divisas. Para lo que resta de la década de los noventa, las políticas agrícolas de la región centroamericana se ven enfocadas a elevar la producción tanto de cultivos ornamentales como de cultivos alimenticios, estando la caña de azúcar como cultivo promisorio.

En Caña de Azúcar la biotecnología puede producir nuevos híbridos, conservar germoplasma, micropropagar cultivares importantes, entre otros; sin embargo, el cultivo in vitro presenta ciertas limi-tantes como: la contaminación bacteriana y fungosa, además la fenolización u oxidación del material vegetal. Las primeras dos dependen directamente del material vegetal y manipulación al momento de la siembra in vitro y la tercera depende de la composición genética del material , la etapa fisiológica y del tipo de explante con que se trabaje (Roca y Mroginski 1991).

Debido a estas limitantes, se realizó el estudio con el objetivo de evaluar la respuesta de las variedades Mex

\footnotetext{
1 Presentado en la XLI Reunión Anual del PCCMCA en El Salvador, Centroamérica. 27 al 31 de marzo, 1996.

2 Universidad Católica de Occidente (UNICO), Santa Ana, El Salvador.
} 
70-485 y Mex 68-P-23 ante la propagación in vitro utilizando distintas modificaciones del medio de Murashige y Skoog, 1962.

\section{MATERIALES Y METODOS}

Los cultivos se mantuvieron a una temperatura promedio de $28^{\circ} \mathrm{C}$. En la primera semana el explante se mantuvo en oscuridad total para reducir la oxidación y estimular la formación de "callo". Luego se expuso a un fotoperiodo de 16/8 horas de luz/oscuridad, con una intensidad lumínica promedio de 2000 lux.

La presente investigación se realizó durante el año de 1995, en el Laboratorio de Cultivo de Tejidos Vegetales, de la Universidad Católica de Occidente, en la ciudad de Santa Ana, El Salvador.

El material vegetal se obtuvo de la cooperativa Talcomunca, ubicada en el cantón Talcomunca, jurisdicción de Nahuizalco, Sonsonate se seleccionaron las variedades Mex 70-485 y Mex 68-P-23(Catálogo de variedades de caña de azúcar, 1991). El material que se ocupó como explante en cultivo de tejidos se obtuvo de plantas de seis a ocho meses de edad.

La porción apical que contenía las hojas jóvenes tuvo un tamaño de 5 a 6 centímetros de longitud. Se pasó a la cámara de flujo laminar, esterilizándola por dos minutos en alcohol al 90\%, con agitación manual continua, se hizo la disección sobre cuadros de papel de $100 \mathrm{~cm}^{2}$ (esterilizados previamente), usando pinza y bisturí.

El procedimiento para la extracción del explante de hojas jóvenes en tallo de caña de azúcar, fue de la siguiente manera:

a. se tomó el material joven y de crecimiento activo.

b. se eliminaron tejidos, hasta dejar la última hoja.

c. posteriormente se eliminó el último entrenudo y se tomó de 5 a 7 explantes de $5 \mathrm{~mm}$ cada uno .
Cuadro 1. Composición del medio de cultivo para la callogénesis, Fase I.

\begin{tabular}{ll}
\hline Componentes & Cantidad \\
\hline Macronutrimentos(ml/1.) & $20^{*}$ \\
Micronutrimentos (ml/1.) & $10^{*}$ \\
Sacarosa (g/l.) & 20 \\
Mioinositol (mg/l.) & 100 \\
Tiamina (mg/l.) & 1.0 \\
2,4-D (mg/l.) & 3.0 \\
\hline
\end{tabular}

* Las concentraciones de las soluciones madres utilizadas, son las recomendadas por Murashige y Skoog en 1996.

Cada explante se colocó en un frasco de $125 \mathrm{cc}$, con $20 \mathrm{ml}$, de medio de cultivo, se selló con plastic wrap. El medio de cultivo que se utilizó fue el de Murashige y Skoog, modificado para cada una de las etapas (Cuadros 1 y 2).

Para la preparación del medio de cultivo, se utilizaron soluciones madres de macro y micronutrimientos. E 1 medio se preparó a partir de Na2-EDTA.2H2O y de FeSO4.7H2O. Los medios de cultivo se solidificaron con 1,7 g/l de Phytagel,( SIGMA), y se ajusto el pH 5,7 con $\mathrm{NaOH} 1 \mathrm{~N}$

Luego de formadas los callos, estos se transfirieron a medios nutritivos MS, con distintas concentraciones de 2,4-D $(3,0 ; 2,5$ y $2,0 \mathrm{mg} / \mathrm{l})$, para el crecimiento y desarrollo de los mismos.

Luego los callos de los distintos tratamientos con 2,4-D fueron puestos en medio MS sin reguladores de crecimiento para la diferenciación de tejidos y obtención de plántulas,( Fase II).

Cuadro 2. Composición del medio de cultivo para el enraizamiento, Fase III.

\begin{tabular}{|c|c|c|c|c|}
\hline Componentes & Medio MS* & Medio ${ }^{1 /}{ }_{2} \mathbf{M S}^{* *}$ & Medio + 5mg/l & AIA* \\
\hline Macronutrimentos $(\mathrm{ml} / \mathrm{l})$ & 20 & 10 & 20 & \\
\hline Micronutrimentos(ml/l) & 10 & 10 & 10 & \\
\hline Sacarosa $(g / l)$ & 20 & 20 & 20 & \\
\hline Mioinositol (mg/l) & 100 & 100 & 100 & \\
\hline Tiamina (mg/l) & 1.0 & 1.0 & 1,0 & \\
\hline AIA (mg/l) &.-- &.-- & 5,0 & \\
\hline
\end{tabular}

* Las concentraciones de las soluciones madres utilizadas, son las recomendadas por Murashige y Skoog en 1996.

** La concentración de los macronutrientes se redujo a la mitad de la recomendada por Murashige y Skoog en 1962. 
Para evaluar el enraizamiento de los brotes, estos fueron transferidos a distintos medios de enraizamiento(Cuadro 2).

El diseño estadístico utilizado fue el irrestricto al azar. Los experimentos se dividen en tres etapas.

\section{Etapa I:}

Dos variedades, Mex 70-485 y Mex 68-P-23, con diez repeticiones. Se evaluaron la formación, crecimiento y proliferación de callo.

\section{Etapa II:}

Con la variedad Mex 70-485. Se compraron tres contracciones de 2,4D : 3,0;2,5 y 2,0 mg/l cada tratamiento tenía diez repeticiones. Se evaluaron el número de plántulas y tamaño de las mismas.

\section{Etapa III:}

Para el enraizamiento de la variedad Mex 70-485, se evaluaron tres tratamientos: MS completo , $1 / 2 \mathrm{MS}(1 / 2 \mathrm{de}$ macronutrimientos y micronutrimientos completos) y MS completo más $5 \mathrm{mg} / \mathrm{l}$ de AIA. Cada tratamiento con diez repeticiones. Se evaluaron directamente el número promedio de raíces y tamaño de raíces y plántulas. En la figura 1 , se muestra el esquema de multiplicación de caña de azúcar empleado en el presente estudio.

\section{RESULTADOS Y DISCUSIÓN}

\section{Callogénesis en las variedades Mex 68-P-23 y Mex 70-485.}

Los "callos" se empezaron a formar a partir de la tercera semana, en los bordes del explante de hoja. A partir de esta semana en la variedad Mex 70-485 se obtuvo en promedio una mayor formación de "callo" con respecto a la variedad Mex 68-P-23, siendo estas diferencias altamente significativas $(\mathrm{P} \leq 0,01)$.

La coloración presentada por lo callos la variedad Mex 70-485 fue de puntos rojos a café, característico de

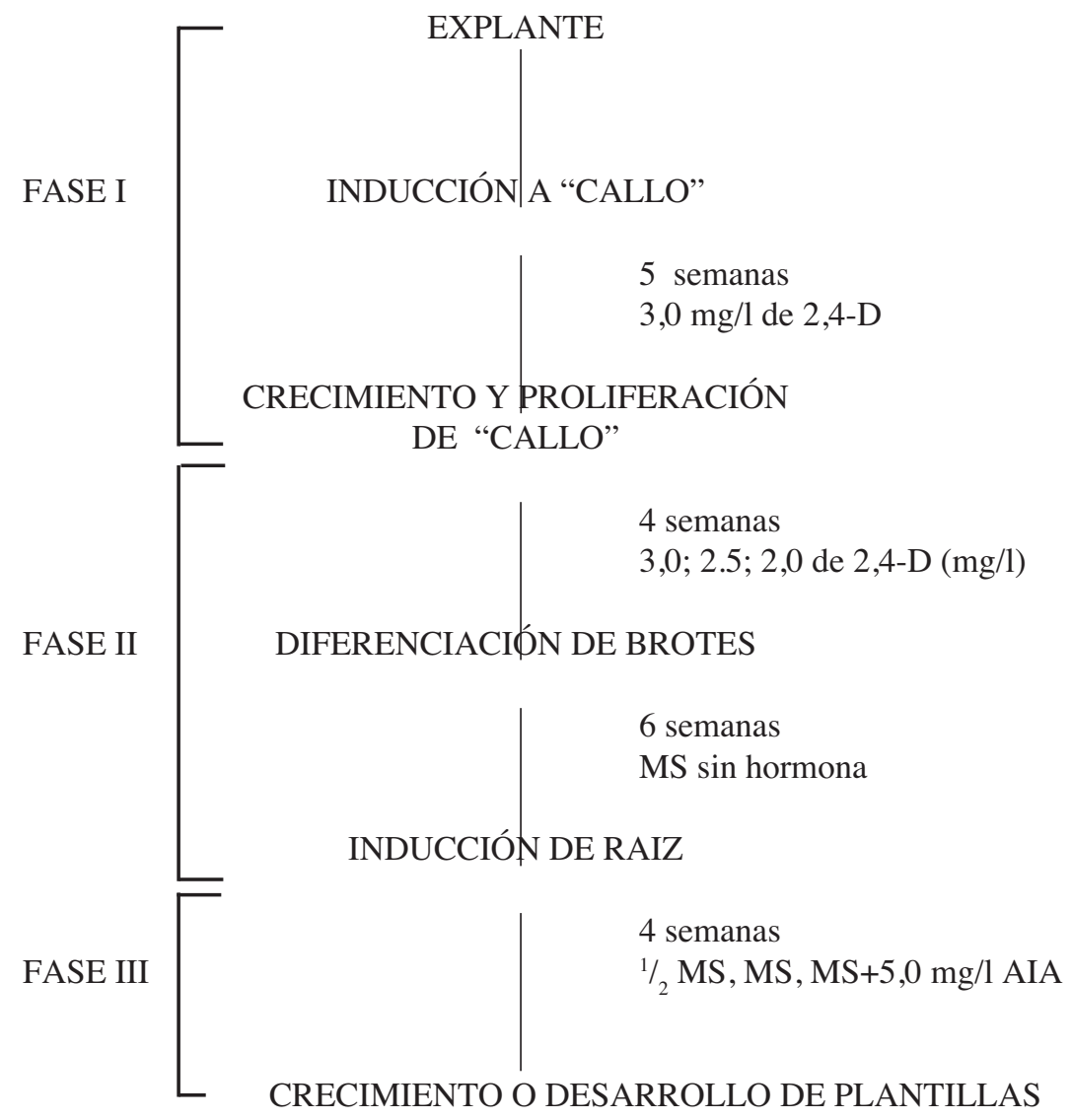

Fig. 1. Esquema de la multiplicación de Caña de Azúcar in vitro, utilizada en el presente estudio. Santa Ana, 1995. 
la variedad en su fase adulta y la variedad Mex 68-P-23 presentó color crema al inicio y luego café oscuro debido a la fenolización.

Es notable la diferencia entre las variedades Mex 70485 y la Mex 68-P-23 en la secreción de fenoles, donde la Mex 68-P-23 mostró mayor secreción y posiblemente causó la inhibición del crecimiento del callo (Thorpe, 1981). Los tejidos meristemáticos poseen alta concentración de polifenoles y éstos causan oxidación e inducen a la formación de quinonas. Las quinonas son conocidas como tóxicas la oxidación inhibe el crecimiento del tejido (Thorpe , 1981) razón por la cual, la Mex 70485 sea probablemente susceptible al ataque de "Barrenador" (Diatraea saccharalis) y la Mex 68-P-23 resistente. Parece entonces, que el grado de producción de fenoles depende posiblemente de la variedad con que se trabaje y no del medio de cultivo que se utilice, como mencionan Roca y Mroginski (1991) al utilizar diferentes medios de cultivo.
La dosis de 3mg/l de 2,4-D indujo la callogénesis en las variedades evaluadas. Esta dosis es recomendada por Heinz y Mee (1969), Payan y Tarcon (1977) y Villalobos (1986) para otras variedades.

Efecto de distintas concentraciones $2,4-\mathrm{D}(3,0 ; 2,5 ; 2,0$ mg/l) en el crecimiento y desarrollo de los callos.

\section{Variedad Mex 68-P-23}

Todos los callos desarrollados transferidos a diferentes concentraciones de 2,4-D, se oxidaron y no crecieron, muriendo posteriormente, lo cual confirma lo mencionado anteriormente, la oxidación depende en gran medida de la variedad en estudio,por lo que no se continuó con las observaciones de la variedad. (Cuadro 3).

\section{Variedad Mex 70-485.}

Se observó crecimiento del callo con mayor proporción en la concentración de 3,0 mg/l de 2,4-D, que en las dos restantes, aún cuando la misma no fue

Cuadro 3. Promedio de formación de "Callo" en las Variedades Mex 68-P-23 y Mex 70-485, Santa Ana, 1995*.

\begin{tabular}{ccc}
\hline \multicolumn{2}{c}{$\begin{array}{c}\text { Promedio de Formación de "Callo" Por explante } \\
\text { Mex 68-P-23 }\end{array}$} & $\begin{array}{c}\text { Mex 70-485 Unidades } \\
\text { Semanas }\end{array}$ \\
\hline 1 & 0,00 & 0,00 \\
2 & 0,00 & 0,00 \\
3 & 0,50 & 1,55 \\
4 & 0,65 & 3,20 \\
5 & 0,65 & 4,25
\end{tabular}

*/ Diferencia significativa . $\mathrm{P}<0.01$ entre variedades.

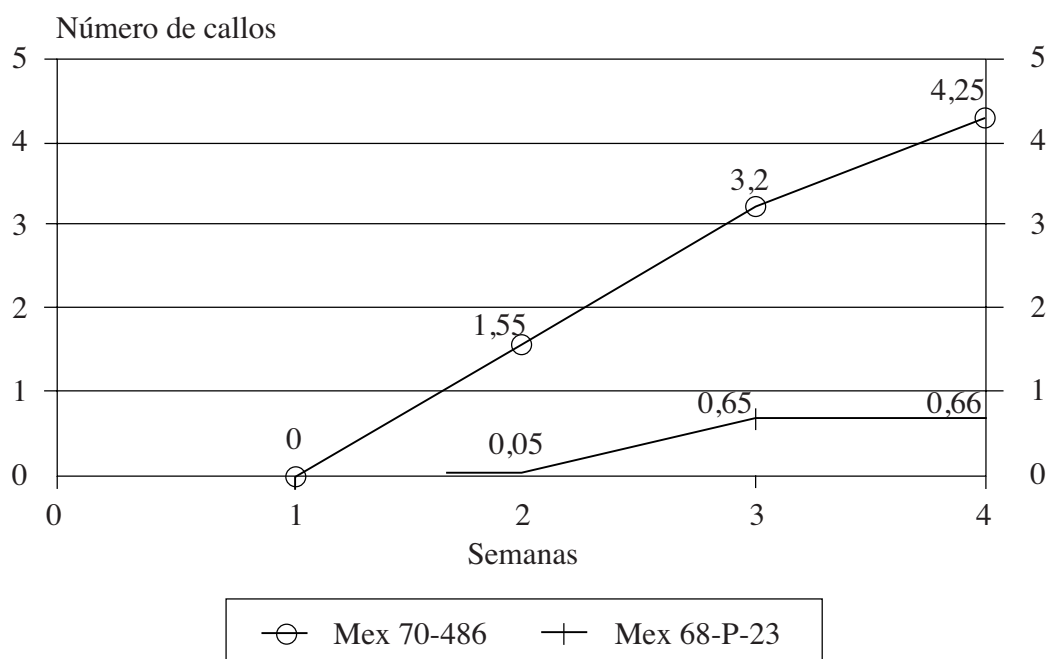

Fig. 2. Formación de "callos" en medio MS con $3.0 \mathrm{mg} / \mathrm{l}$ de 2,4-D de las variedades Mex 68-P-23 y Mex 70-485. Propagación in vitro de Caña de Azúcar, Santa Ana, 1995. 
significativa.La formación de callo se midió a partir de los puntos de crecimiento originados en el explante. (Cuadro 3).

Se puede observar que los "callos" transferidos a medio MS con diferentes concentraciones de 2,4-D, no diferenciaron brotes según recomendación de Payan y Tarcon (1977). Sólo hubo crecimiento de los "callos", confirmándose la función del 2,4-D es la de inducir la división y alargamiento celular.

\section{Diferenciación de tejidos de la variedad Mex 70-485.}

Los “callos" tratados con 3,0; 2,5 y 2.0 mg/l. de 2,4D fueron transferidos a medio MS sin reguladores de crecimiento. Los "callos" crecieron mostrando coloraciones blancas y de color café claro. Durante la cuarta y quinta semana se observó puntos verdes, los cuales son los primeros órganos diferenciados.

A la sexta semana fueron nuevamente transferidos a medio MS sin reguladores de crecimiento con el objetivo de mantener vivo el tejido. Los "callos" procedentes del tratamiento con 2,0 mg/l de 2,4-D se oxidaron totalmente , aparentemente la concentración de 2,4-D no fue suficiente para continuar un crecimiento vigoroso en el "callo"; concecuentemente, al ser transferidos al medio MS sin reguladores de crecimiento la diferenciación fue muy pobre posteriomente muriendo, por lo que no se continuó con las observaciones de este tratamiento.

Después de once semanas se pudo obtener los siguientes datos (Cuadro 4).
En el análisis realizado se obtuvieron diferencias significativas en lo que respecta al promedio de brotes, obteniéndose el mayor número al utilizar la concentración de 3,0 mg/l para el crecimiento de callo $(\mathrm{P} \leq 0,05)$.

De igual manera se analizó el tamaño de los brotes.Y se observaron diferencias significativas entre las concentraciones de 2,4-D utilizadas para el crecimiento del "callo" (Cuadro 5) $(\mathrm{P} \leq 0,05)$.

La diferenciación se dio al someter los callos a medio MS sin reguladores de crecimiento tal y como lo menciona Heinz y Mee (1969). Aun cuando los callos procedentes de los distintos tratamientos con 2,4-D (Cuadro 4) no diferenciaron de igual forma, posiblemente fue por la adaptabilidad al mismo ácido. La literatura no cita claramente qué efecto puede causar las distintas concentraciones del 2,4-D, asumiendo que la mejor concentración en la formación de callo fue 3,0 mg/l por consiguiente al pasarlo a medio MS sin reguladores de crecimiento habría una mayor formación de brotes por callo (Cuadro 5).

Cuadro 4. Promedio de brotes por callo provenientes de medio MS con distintas concentraciones de 2,4-D de la variedad Mex 70-485, Santa Ana, 1995*.

\begin{tabular}{cc}
\hline $\begin{array}{c}\text { Concentración de } \\
\text { 2,4-D (mg/L) }\end{array}$ & $\begin{array}{c}\text { Promedio de brotes } \\
\text { por "callo" }\end{array}$ \\
\hline 3,0 & 8,20 \\
2,5 & 1,10 \\
\hline * & Diferencias significativas /P $\leq 0.05$ entre concentraciones \\
de 2,4- D.
\end{tabular}

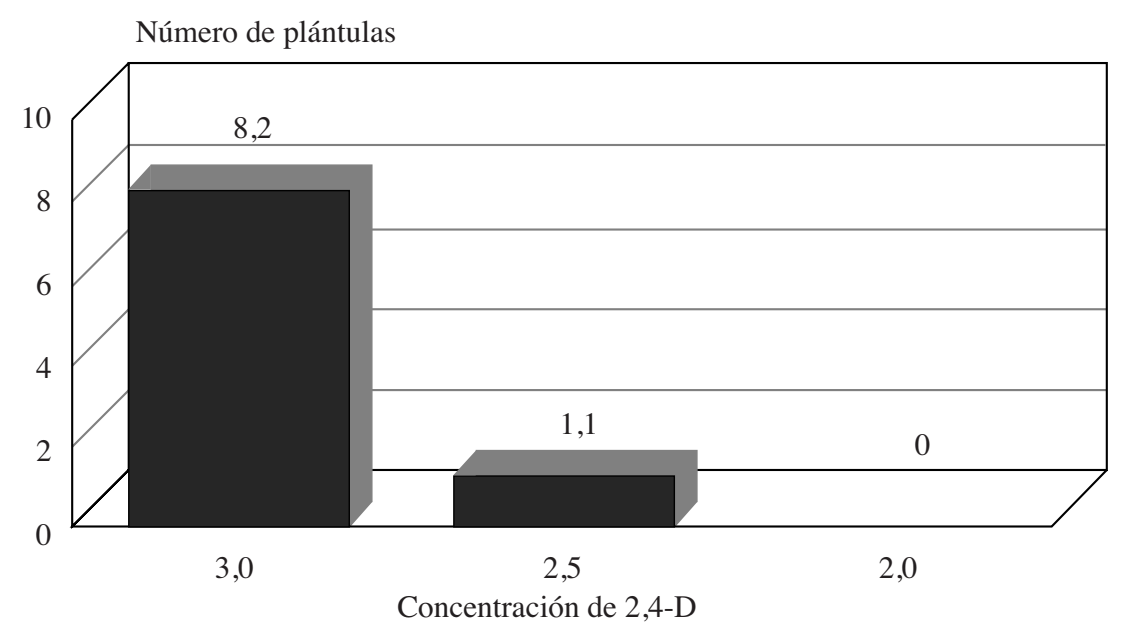

Fig. 3. Número de brotes formadas en medio MS sin reguladores de crecimiento en la Variedad Mex 70-485, provenientes de medio MS con distintas concentraciones de 2,4-D, Santa Ana, 1995. 
Cuadro 5. Promedio del tamaño de plántulas de la variedad Mex 70-485, Santa Ana, 1995.

\begin{tabular}{cc}
\hline $\begin{array}{c}\text { Concentración de } \\
\mathbf{2 , 4 - D}(\mathbf{m g} / \mathbf{l})\end{array}$ & $\begin{array}{c}\text { Promedio de tamaño } \\
\text { de plántulas }(\mathbf{c m})\end{array}$ \\
\hline 3,0 & 2,67 \\
2,5 & 0,65 \\
\hline
\end{tabular}

$\mathrm{P}=0,05$

Efecto del AIA y concentración de Macronutrimentos en la rizogénesis

De las plántulas obtenidas del tratamiento con 3,0 $\mathrm{mg} / \mathrm{l}$ de 2,4-D,se escogieron plantas bien formadas para pasarlas a un medio de enraizamiento. Estas plantas tenían tallos individuales y se les retira los tejidos necrosados de consistencia dura y coloración oscura que se acumularon alrededor del cuello durante la fase de diferenciación.

Luego de la cuarta semana en medio de enraizamiento, se obtuvo diferencias altamente significativas (Cuadro 6) $(\mathrm{P} \leq 0,01)$.

En análisis realizado entre el número de raíces y el tamaño de las raíces. Existe una correlación negativa (inversamente proporcional; $r=-0,098$ ) entre el número de raíces y el tamaño de éstas.La correlación entre el número de raíces y el tamaño de la planta no fue significativa (Cuadro 7) $(r=0,421)$.
Los tratamientos realizados con 1/2 MS, MS, $5 \mathrm{mg} /$ 1 de AIA, todos produjeron raíces tal como lo menciona Thorpe (1981); sin embargo, el mejor medio es el que contiene AIA como menciona Roca y Mronginski (1991).

Cuadro 6. Promedio de formación de raíces por Plántula. Variedad Mex 70-485, Santa Ana, 1995.

\begin{tabular}{cc}
\hline $\begin{array}{c}\text { Medio de } \\
\text { enraizamiento }\end{array}$ & $\begin{array}{c}\text { Promedio de raíces } \\
\text { de plántulas }\end{array}$ \\
\hline $1 / 2$ MS & 4,10 \\
MS & 2,80 \\
MS + AIA & 9,40 \\
\hline
\end{tabular}

*/ Diferencias significativas al $\mathrm{P}=0,01$ entre dos medios de enraizamiento.

Los medios 1/2MS y MS en particular produjeron raíces muy filamentosas confirmando lo dicho por Murashige (1962) citado por Roca y Mroginski (1991).

Hay que recalcar lo expresado por Roca y Mroginski (1991) en donde mencionan que el escaso enraizamiento es más frecuente en plantas provenientes de micropropagación a causa de las altas concentraciones de citocininas. Hay que recordar que las plantas enraizadas en este estudio no fueron micropropagadas, lo cual pudo haber favorecido el enraizamiento.

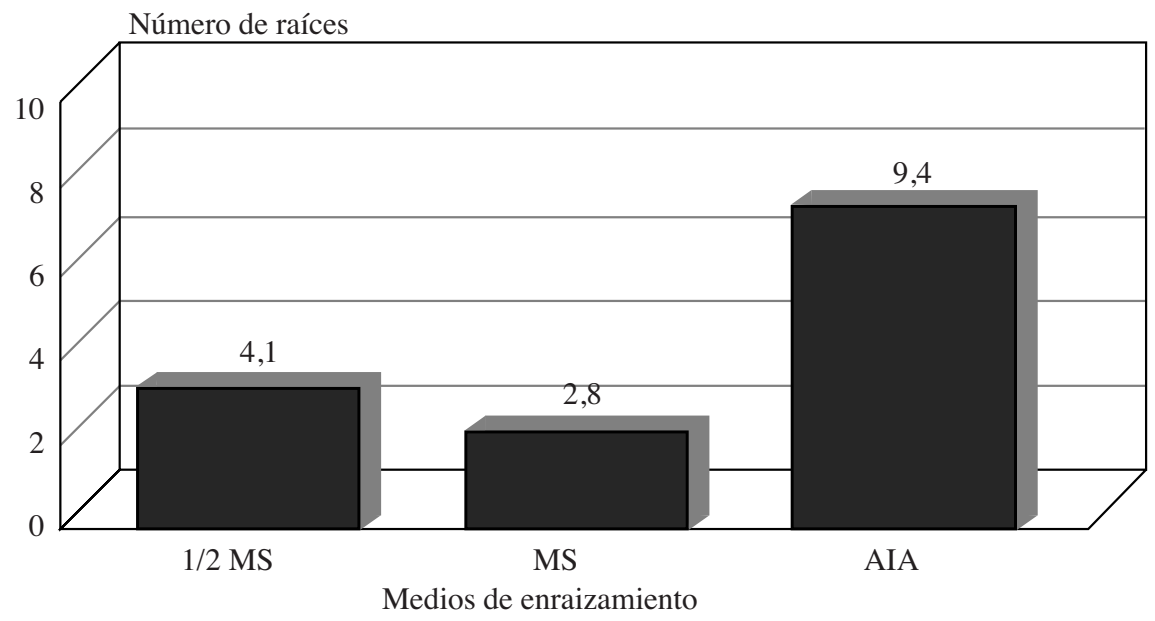

Fig 4. Número de raíces producidas por planta de la Variedad Mex 70-485 en distintos medios de enraizamiento, Santa Ana, 1995. 
Cuadro 7. Relación entre el tamaño de raíces y el tamaño de plantas en la Variedad Mex 70-485, Santa Ana, 1995.

\begin{tabular}{ccc}
\hline $\begin{array}{c}\text { Medio de } \\
\text { enraizamiento }\end{array}$ & $\begin{array}{c}\text { Tamaño de } \\
\text { raíces }(\mathbf{c m})\end{array}$ & $\begin{array}{c}\text { Tamaño de } \\
\text { plantas }(\mathbf{c m})\end{array}$ \\
\hline 1/2 MS & 3,47 & 11,62 \\
MS & 3,96 & 12,79 \\
MS+AIA & 3,68 & 12,69 \\
\hline
\end{tabular}

*/ Diferencias no significativas entre los medios de enraizamiento.

\section{LITERATURA CITADA}

CATALOGO DE VARIEDADES DE CAÑA DE AZUCAR.

1991. Compañía azucarera salvadoreña. Departamento agrícola, Centro experimental y capacitación. El Salvador. $63 \mathrm{p}$.
HEINZ, D.J.; MEE, G. 1969. Plant differentiation from callus tissue of Saccharum species. Crop. Sci. 9:346-348.

MURASHIGE, T.; SKOOG, F. 1962. A revised medium for rapid growth and bioassays with tobacco tissue culture. Physiol. Plant. 15:437-497.

PAYAN, F.C.; TARCON, G. 1977. Técnicas para la micropropagación de la caña de azúcar mediante el cultivo de tejido y de yemas axilares. Acta Agronómica. $27(1 / 4)$.

ROCA, M.W.; MROGINSKI, L.A. 1991. Cultivo de Tejidos en la Agricultura: Fundamentos y aplicaciones. CIAT (Centro Internaciol de Agricultura Tropical). Cali, Colombia. p. $542-575$.

THORPE, T. A. 1981. Plant tissue culture methods and applications in agriculture. Academic Press, Nueva York.

VILLALOBOS R, I. M. 1986. Producción de somaclones en tres cultivos comerciales de caña de azúcar (Saccharum spp.) a partir de "callo"s inducidos in vitro Tesis Ing. Agr. Universidad de Costa Rica. C. R. 